MATHEMATICS OF COMPUTATION

Volume 72, Number 244, Pages 1577-1595

S 0025-5718(02)01502-8

Article electronically published on December 18, 2002

\title{
REGULARITY ESTIMATES \\ FOR ELLIPTIC BOUNDARY VALUE PROBLEMS IN BESOV SPACES
}

\author{
CONSTANTIN BACUTA, JAMES H. BRAMBLE, AND JINCHAO XU
}

\begin{abstract}
We consider the Dirichlet problem for Poisson's equation on a nonconvex plane polygonal domain $\Omega$. New regularity estimates for its solution in terms of Besov and Sobolev norms of fractional order are proved. The analysis is based on new interpolation results and multilevel representations of norms on Sobolev and Besov spaces. The results can be extended to a large class of elliptic boundary value problems. Some new sharp finite element error estimates are deduced.
\end{abstract}

\section{INTRODUCTION}

Regularity estimates for the solutions of elliptic boundary value problems in terms of Sobolev norms of fractional order are known as shift theorems or shift estimates. The shift estimates for the Laplace operator with Dirichlet boundary conditions on nonsmooth domains are well known (see, e.g., 18], 20], 24]). The classical regularity estimate for the case when $\Omega$ is a nonconvex polygonal domain in $\mathbb{R}^{2}$, with boundary $\partial \Omega$, and the largest re-entrant corner of measuure $\omega$ is as follows:

If $u$ is the variational solution of

$$
\left\{\begin{aligned}
-\Delta u=f & \text { in } \quad \Omega, \\
u=0 & \text { on } \quad \partial \Omega,
\end{aligned}\right.
$$

then, for $0<s<s_{0}=\pi / \omega$, we have

$$
\|u\|_{1+s} \leq C(s)\|f\|_{-1+s}, \quad \text { for all } f \in H^{-1+s}(\Omega) .
$$

Here the norms involved are the standard Sobolev fractional norms.

We will prove that, for the critical case $s=s_{0}$, the following estimate holds:

$$
\|u\|_{B^{1+s_{0}(\Omega)}} \leq c\|f\|_{B_{01}^{-1+s_{0}}(\Omega)}, \quad \text { for all } f \in B_{01}^{-1+s_{0}}(\Omega),
$$

where $B^{1+s_{0}}(\Omega)$ is a standard Besov space and $B_{01}^{-1+s_{0}}(\Omega)$, defined in Section 3, is also a Besov type space of order $-1+s_{0}$ which contains all the Hilbert spaces $H^{-1+s}$ with $s>s_{0}$. The new shift result leads to new finite element convergence estimates.

Received by the editor January 26, 2002 and, in revised form, March 25, 2002.

2000 Mathematics Subject Classification. Primary 65N30, 46B70, 35J67, 35J05.

Key words and phrases. Interpolation spaces, finite element method, multilevel decomposition, shift theorems, Besov spaces.

The work of the second author was supported in part under NSF Grant No. DMS-9973328.

The work of the third author was supported under NSF Grant No. DMS-0074299. 
The method presented here can be extended to other boundary conditions such as Neumann or mixed Neumann-Dirichlet conditions.

In this paper, the technique involved in proving shift results is the real method of interpolation of Lions and Peetre ([3], [21] and [22]), the cases $p=2$ and $p=\infty$ (where $p$ is the second Besov index), combined with the subspace interpolation theory. We are led to the following type of interpolation problem. If $X$ and $Y$ are Sobolev spaces of integer order and $X_{K}$ is a subspace of codimension one of $X$, then how can one characterize the interpolation spaces between $X_{K}$ and $Y$ for $p=\infty$ ? The problem was studied in [18], [17] and [2] for $p=2$ and particular spaces $X$ and $X_{K}$. In those papers the intermediate spaces are the standard Sobolev (Hilbert) spaces, while in this paper, we will focus on the non-Hilbert space case $p=\infty$ and the critical value $s=s_{0}$.

The interpolation results presented in Section 2 give a natural formula connecting the norms on the intermediate subspaces $\left[X_{K}, Y\right]_{s, \infty}$ and $[X, Y]_{s, \infty}$ when $X_{K}$ is of codimension one. Our approach in proving subspace interpolation problems is to use multilevel representations of the norms for the Sobolev spaces of integer order. The Besov norms obtained by interpolation (the case $p=\infty$ ) between Sobolev spaces of integer order have good multilevel representations. (For multilevel representations of norms see, e.g., [8], [10] and [25].) In Section 3 the main result concerning the codimension-one subspace interpolation problem is presented. Shift theorems for the Poisson equation on polygonal domains are considered in Section 4 In the last section, a straightforward application of the above interpolation results is shown to lead to some new estimates for finite element approximations.

\section{INTERPOLATION RESULTS}

In this section we give some basic definitions and results concerning Besov spaces and Hilbert spaces as interpolation between Hilbert spaces, using the real method of interpolation of Lions and Peetre (see [3], 4], 21]).

2.1. Interpolation between Hilbert spaces. Let $X, Y$ be separable Hilbert spaces with inner products $(\cdot, \cdot)_{X}$ and $(\cdot, \cdot)_{Y}$ respectively, and satisfying, for some positive constant $c$,

$$
\left\{\begin{array}{l}
X \text { is a dense subset of } Y \text { and } \\
\|u\|_{Y} \leq c\|u\|_{X} \quad \text { for all } u \in X,
\end{array}\right.
$$

where $\|u\|_{X}^{2}=(u, u)_{X}$ and $\|u\|_{Y}^{2}=(u, u)_{Y}$.

Let $D(S)$ denote the subset of $X$ consisting of all elements $u$ such that the map

$$
v \rightarrow(u, v)_{X}, v \in X,
$$

is continuous in the topology induced by $Y$.

For any $u$ in $D(S)$ the anti-linear form (2.2) can be uniquely extended to a continuous anti-linear form on $Y$. Then, by the Riesz representation theorem, there exists an element $S u$ in $Y$ such that

$$
(u, v)_{X}=(S u, v)_{Y} \quad \text { for all } v \in X .
$$

In this way $S$ is a well defined operator with domain $D(S)$ in $Y$. The next result illustrates the properties of $S$ (see [21]). 
Proposition 2.1. The domain $D(S)$ of the operator $S$ is dense in $X$, and consequently $D(S)$ is dense in $Y$. The operator $S: D(S) \subset Y \rightarrow Y$ is a bijective, selfadjoint and positive definite operator. The inverse operator $S^{-1}: Y \rightarrow D(S) \subset Y$ is a bounded symmetric positive definite operator and

$$
\left(S^{-1} z, u\right)_{X}=(z, u)_{Y} \quad \text { for all } z \in Y, u \in X .
$$

The interpolation spaces $[X, Y]_{s, 2}$ and $[X, Y]_{s, \infty}$ for $s \in(0,1)$ are defined using the function $K$, where for $u \in Y$ and $t>0$,

$$
K(t, u):=\inf _{u_{0} \in X}\left(\left\|u_{0}\right\|_{X}^{2}+t^{2}\left\|u-u_{0}\right\|_{Y}^{2}\right)^{1 / 2} .
$$

Then the space $[X, Y]_{s, \infty}$ consists of all $u \in Y$ such that

$$
\sup _{t>0} t^{-2 s} K(t, u)^{2}<\infty,
$$

and $[X, Y]_{s, 2}$ consists of all $u \in Y$ such that

$$
\int_{0}^{\infty} t^{-(2 s+1)} K(t, u)^{2} d t<\infty .
$$

The norm on $[X, Y]_{s, 2}$ is defined by

$$
\|u\|_{[X, Y]_{s, 2}}^{2}:=\mathbf{c}_{s}^{2} \int_{0}^{\infty} t^{-(2 s+1)} K(t, u)^{2} d t,
$$

where

$$
\mathbf{c}_{s}:=\left(\int_{0}^{\infty} \frac{t^{1-2 s}}{t^{2}+1} d t\right)^{-1 / 2}=\sqrt{\frac{2}{\pi} \sin (\pi s)} .
$$

Similarly, the norm on $[X, Y]_{s, \infty}$ is defined by

$$
\|u\|_{[X, Y]_{s, \infty}}:=\sup _{t>0} t^{-s} K(t, u) \text {. }
$$

By definition we take

$$
[X, Y]_{0,2}:=X, \quad[X, Y]_{1,2}:=Y
$$

and

$$
[X, Y]_{0, \infty}:=X, \quad[X, Y]_{1, \infty}:=Y .
$$

The next lemma provides the relation between $K(t, u)$ and the connecting operator $S$.

Lemma 2.1. For all $u \in Y$ and $t>0$,

$$
K(t, u)^{2}=t^{2}\left(\left(I+t^{2} S^{-1}\right)^{-1} u, u\right)_{Y} .
$$

Proof. Using the density of $D(S)$ in $X$, we have

$$
K(t, u)^{2}=\inf _{u_{0} \in D(S)}\left(\left\|u_{0}\right\|_{X}^{2}+t^{2}\left\|u-u_{0}\right\|_{Y}^{2}\right) .
$$

Let $v=S u_{0}$. Then

$$
K(t, u)^{2}=\inf _{v \in Y}\left(\left(S^{-1} v, v\right)_{Y}+t^{2}\left\|u-S^{-1} v\right\|_{Y}^{2}\right) .
$$

Solving the minimization problem (2.6), we obtain that the element $v$ which gives the optimum satisfies

$$
\left(I+t^{2} S^{-1}\right) v=t^{2} u
$$


and

$$
\left(S^{-1} v, v\right)_{Y}+t^{2}\left\|u-S^{-1} v\right\|_{Y}^{2}=t^{2}\left(\left(I+t^{2} S^{-1}\right)^{-1} u, u\right)_{Y} .
$$

Remark 2.1. Lemma 2.1 yields other expressions for the norms on $[X, Y]_{s, 2}$ and $[X, Y]_{s, \infty}$. Namely,

$$
\|u\|_{[X, Y]_{s, 2}}^{2}=\mathbf{c}_{s}^{2} \int_{0}^{\infty} t^{-2 s+1}\left(\left(I+t^{2} S^{-1}\right)^{-1} u, u\right)_{Y} d t,
$$

and

$$
\|u\|_{[X, Y]_{s, \infty}}^{2}=\sup _{t>0} t^{2(1-s)}\left(\left(I+t^{2} S^{-1}\right)^{-1} u, u\right)_{Y} .
$$

2.2. Interpolation between subspaces of a Hilbert space. Let $\mathcal{K}=\operatorname{span}\{\varphi\}$ be a one-dimensional subspace of $X$ and let $X_{\mathcal{K}}$ be the orthogonal complement of $\mathcal{K}$ in $X$ in the $(\cdot, \cdot)_{X}$ inner product. We are interested in determining the Besov interpolation spaces of $X_{\mathcal{K}}$ and $Y$, where on $X_{\mathcal{K}}$ we consider again the $(\cdot, \cdot)_{X}$ inner product. To apply the interpolation results from the previous section, we need to check that the density part of the condition (2.1) is satisfied for the pair $\left(X_{\mathcal{K}}, Y\right)$.

For $\varphi \in \mathcal{K}$, define the linear functional $\Lambda_{\varphi}: X \rightarrow C$, by

$$
\Lambda_{\varphi} u:=(u, \varphi)_{X}, u \in X .
$$

The following result is an extension of Kellogg's lemma [18. The proof can be found in 2]. For completeness we include the proof also.

Lemma 2.2. Let $\mathcal{K}$ be a closed subspace of $X$ and let $X_{\mathcal{K}}$ be the orthogonal complement of $\mathcal{K}$ in $X$ in the $(\cdot, \cdot)_{X}$ inner product. The space $X_{\mathcal{K}}$ is dense in $Y$ if and only if the following condition is satisfied:

$$
\left\{\begin{array}{l}
\Lambda_{\varphi} \text { is not bounded in the topology of } Y \\
\quad \text { for all } \varphi \in \mathcal{K}, \varphi \neq 0 .
\end{array}\right.
$$

Proof. First let us assume that the condition (2.9) does not hold. Then for some nonzero $\varphi \in \mathcal{K}$ the functional $\Lambda_{\varphi}$ is a bounded functional in the topology induced by $Y$. Thus, the kernel of $\Lambda_{\varphi}$ is a closed subspace of $X$ in the topology induced by $Y$. Since $X_{\mathcal{K}}$ is contained in $\operatorname{Ker}\left(\Lambda_{\varphi}\right)$ it follows that

$$
{\overline{X_{\mathcal{K}}}}^{Y} \subset{\overline{\operatorname{Ker}\left(\Lambda_{\varphi}\right)}}^{Y}=\operatorname{Ker}\left(\Lambda_{\varphi}\right) .
$$

Hence $X_{\mathcal{K}}$ fails to be dense in $Y$.

Conversely, assume that $X_{\mathcal{K}}$ is not dense in $Y$; then $Y_{0}={\overline{X_{\mathcal{K}}}}^{Y}$ is a proper closed subspace of $Y$. Let $y_{0} \in Y$ be in the orthogonal complement of $Y_{0}$, and define the linear functional $\Psi: Y \rightarrow C$ by

$$
\Psi u:=\left(u, y_{0}\right)_{Y}, u \in Y .
$$

$\Psi$ is a continuous functional on $Y$. Let $\psi$ be the restriction of $\Psi$ to the space $X$. Then $\psi$ is a continuous functional on $X$. By the Riesz representation theorem, there is $v_{0} \in X$ such that

$$
\left(u, v_{0}\right)_{X}=\left(u, y_{0}\right)_{Y}, \quad \text { for all } u \in X .
$$

Let $P_{\mathcal{K}}$ be the $X$ orthogonal projection onto $\mathcal{K}$ and take $u=\left(I-P_{\mathcal{K}}\right) v_{0}$ in (2.10).

Since $\left(I-P_{\mathcal{K}}\right) v_{0} \in X_{\mathcal{K}}$, we have $\left(\left(I-P_{\mathcal{K}}\right) v_{0}, y_{0}\right)_{Y}=0$ and

$$
0=\left(\left(I-P_{\mathcal{K}}\right) v_{0}, v_{0}\right)_{X}=\left(\left(I-P_{\mathcal{K}}\right) v_{0},\left(I-P_{\mathcal{K}}\right) v_{0}\right)_{X} .
$$


It follows that $v_{0}=P_{\mathcal{K}} v_{0} \in \mathcal{K}$ and, via (2.10), that $\psi=\Lambda_{v_{0}}$ is continuous in the topology of $Y$. This is exactly the opposite of (2.9), and the proof is complete.

For the remaining part of this section we assume that $\Lambda_{\varphi}$ is not bounded in the topology of $Y$, so the condition (2.1) is satisfied for the pair $\left(X_{\mathcal{K}}, Y\right)$. We denote $X_{\mathcal{K}}$ by $X_{\varphi}$. It follows from the previous section that the operator $S_{\varphi}: D\left(S_{\varphi}\right) \subset Y \rightarrow Y$ defined by

$$
(u, v)_{X}=\left(S_{\varphi} u, v\right)_{Y} \quad \text { for all } v \in X_{\varphi}
$$

has the same properties as $S$. Consequently, the norm on the intermediate space $\left[X_{\varphi}, Y\right]_{s, \infty}$ is given by

$$
\|u\|_{\left[X_{\varphi}, Y\right]_{s, \infty}}^{2}:=\sup _{t>0} t^{2(1-s)}\left(\left(I+t^{2} S_{\varphi}^{-1}\right)^{-1} u, u\right)_{Y} .
$$

Our aim in this section is to determine sufficient conditions for $\varphi$ such that the norm $\left[X_{\varphi}, Y\right]_{s, \infty}$ can be compared with more familiar intermediate norms. First, we note that the operators $S_{\varphi}$ and $S$ are related by the following identity:

$$
S_{\varphi}^{-1}=\left(I-Q_{\varphi}\right) S^{-1},
$$

where $Q_{\varphi}: X \rightarrow \mathcal{K}$ is the orthogonal projection onto $\mathcal{K}=\operatorname{span}\{\varphi\}$. The proof of (2.13) follows easily from the definitions of the operators involved.

Next, (2.13) leads to a formula relating the norms on $\left[X_{\varphi}, Y\right]_{s, \infty}$ and $[X, Y]_{s, \infty}$.

Theorem 2.1. For any $u \in X$ we have

$$
\|u\|_{\left[X_{\varphi}, Y\right]_{s, \infty}}^{2}=\sup _{t>0}\left(t^{2-2 s}(u, u)_{Y, t}+t^{4-2 s} \frac{\left|(u, \varphi)_{Y, t}\right|^{2}}{(\varphi, \varphi)_{X, t}}\right) .
$$

In particular, for $u \in X_{\varphi}$ we have

$$
\|u\|_{\left[X_{\varphi}, Y\right]_{s, \infty}}^{2}=\sup _{t>0}\left(t^{2-2 s}(u, u)_{Y, t}+t^{-2 s} \frac{\left|(u, \varphi)_{X, t}\right|^{2}}{(\varphi, \varphi)_{X, t}}\right),
$$

where

$$
(u, v)_{X, t}:=\left(\left(I+t^{2} S^{-1}\right)^{-1} u, v\right)_{X} \quad \text { for all } u, v \in X,
$$

and

$$
(u, v)_{Y, t}:=\left(\left(I+t^{2} S^{-1}\right)^{-1} u, v\right)_{Y} \quad \text { for all } u, v \in Y .
$$

Proof. Let $u$ be fixed in $X$, and set

$$
w:=\left(I+t^{2} S^{-1}\right)^{-1} u \text { and } w_{\varphi}:=\left(I+t^{2} S_{\varphi}^{-1}\right)^{-1} u .
$$

Then the norms on $[X, Y]_{s, \infty}$ and $\left[X_{\varphi}, Y\right]_{s, \infty}$ are given by

$$
\|u\|_{[X, Y]_{s, \infty}}=\sup _{t>0} t^{1-s}(w, u)_{Y}^{1 / 2}
$$

and

$$
\|u\|_{\left[X_{\varphi}, Y\right]_{s, \infty}}=\sup _{t>0} t^{1-s}\left(w_{\varphi}, u\right)_{Y}^{1 / 2}
$$

respectively.

From (2.18) it follows that

$$
\left(I+t^{2} S_{\varphi}^{-1}\right) w_{\varphi}=u
$$


Combining (2.13) and (2.21), we obtain

$$
\left(I+t^{2} S^{-1}\right) w_{\varphi}=u+t^{2} \alpha \varphi
$$

where $\alpha$ is a constant determined by $Q_{\varphi}\left(S^{-1} w_{\varphi}\right)=\alpha \varphi$. Equivalently, applying $\left(I+t^{2} S^{-1}\right)^{-1}$ to both sides, we have

$$
w_{\varphi}=w+t^{2} \alpha\left(I+t^{2} S^{-1}\right)^{-1} \varphi .
$$

We calculate the coefficient $\alpha$ by taking the $(\cdot, \cdot)_{X}$ inner product with $\varphi$ on both sides of (2.22); i.e.,

$$
\left(w_{\varphi}, \varphi\right)_{X}=(w, \varphi)_{X}+t^{2} \alpha\left(\left(I+t^{2} S^{-1}\right)^{-1} \varphi, \varphi\right)_{X} .
$$

From (2.21), since $S_{\varphi}^{-1} w_{\varphi} \in X_{\varphi}$, one sees that $\left(w_{\varphi}, \varphi\right)_{X}=(u, \varphi)_{X}$. With the notation adopted in (2.16) and (2.18) we obtain

$$
t^{2} \alpha=\frac{(u, \varphi)_{X}-(u, \varphi)_{X, t}}{(\varphi, \varphi)_{X, t}} .
$$

Using (2.4) and a simple manipulation of the operator $S$, we get

$$
t^{2}(u, \varphi)_{Y, t}=(u, \varphi)_{X}-(u, \varphi)_{X, t} .
$$

Thus

$$
\alpha=\frac{(u, \varphi)_{Y, t}}{(\varphi, \varphi)_{X, t}} .
$$

Now, going back to (2.22), we get

$$
\left(w_{\varphi}, u\right)_{Y}=(w, u)_{Y}+t^{2} \frac{\left|(u, \varphi)_{Y, t}\right|^{2}}{(\varphi, \varphi)_{X, t}} .
$$

Combining (2.19), (2.20) and (2.24) completes the proof of (2.14). Then, (2.15) follows from (2.14) and (2.23).

Theorem 2.1 can be easily extended to the case in which the dimension of $K$ is finite (see [2]). Such an extension would be needed, for example, to treat the case of mixed Neumann-Dirichlet conditions.

\section{Subspace interpolation by multileVel norms}

Let $\Omega$ be a domain in $\mathbb{R}^{2}$ with boundary $\partial \Omega$. Let $H_{0}^{1}$ denote the space of all functions in $H^{1}(\Omega)$ which vanish on $\partial \Omega$. Assume that

$$
M_{1} \subset M_{2} \subset \cdots \subset M_{k} \subset \cdots
$$

is a sequence of finite dimensional subspaces of $H_{0}^{1}(\Omega)$ whose union is dense in $H_{0}^{1}(\Omega)$, and assume that an equivalent norm on $H_{0}^{1}(\Omega)$ is given by

$$
\|u\|_{1}:=\left(\sum_{k=1}^{\infty} \lambda_{k}\left\|\left(Q_{k}-Q_{k-1}\right) u\right\|^{2}\right)^{1 / 2},
$$

where $Q_{k}$ denotes the $L^{2}(\Omega)$ orthogonal projection onto $M_{k},\|\cdot\|=\|\cdot\|_{L^{2}(\Omega)}, Q_{0}=0$, and $\lambda_{k}=4^{k-1}$. Proofs for the multilevel representation of the norm on $H^{1}$ for specific choices of the spaces $M_{k}$ can be found in [2], [10], [25] and [27] . 
3.1. Scales of multilevel norms. On $H_{0}^{1}(\Omega)$ we consider the norm given by (3.1) and define $H^{-1}(\Omega)$ to be the dual of $H_{0}^{1}(\Omega)$. The elements of $L^{2}(\Omega)$ can be viewed as continuous linear functionals on $H_{0}^{1}(\Omega)$, and we have the natural continuous and dense embeddings

$$
H_{0}^{1}(\Omega) \subset L^{2}(\Omega) \subset H^{-1}(\Omega)
$$

One can easily check that

$$
\|u\|_{H^{-1}(\Omega)}^{2}:=\sum_{k=1}^{\infty} \lambda_{k}^{-1}\left\|\left(Q_{k}-Q_{k-1}\right) u\right\|^{2}, \quad \text { for all } u \in L^{2}(\Omega) .
$$

Further, the inner product on $H^{-1}(\Omega)$ is

$$
(u, v)_{-1}:=\sum_{k=1}^{\infty} \lambda_{k}^{-1}\left(\left(Q_{k}-Q_{k-1}\right) u, v\right), \quad \text { for all } u, v \in L^{2}(\Omega) .
$$

Then the pairs $\left(H_{0}^{1}(\Omega), L^{2}(\Omega)\right)$ and $\left(L^{2}(\Omega), H^{-1}(\Omega)\right)$ satisfy the condition (2.1), and the operator $S$ associated with each of these pairs is given (in both cases) by

$$
S u=\sum_{k=1}^{\infty} \lambda_{k}\left(Q_{k}-Q_{k-1}\right) u, \quad \text { for all } u \in D(S) .
$$

For example, for $X=L^{2}(\Omega)$ and $Y=H^{-1}(\Omega)$, we have

$$
(u, v)_{Y, t}=\sum_{k=1}^{\infty} \frac{1}{\lambda_{k}+t^{2}}\left(\left(Q_{k}-Q_{k-1}\right) u, v\right), \quad u, v \in Y .
$$

and

$$
(u, v)_{X, t}=\sum_{k=1}^{\infty} \frac{\lambda_{k}}{\lambda_{k}+t^{2}}\left(\left(Q_{k}-Q_{k-1}\right) u, v\right), \quad u, v \in X .
$$

For any $s \in[0,1]$, let

$$
H_{0}^{s}(\Omega):=\left[H_{0}^{1}(\Omega), L^{2}(\Omega)\right]_{1-s, 2}, \quad H^{-s}(\Omega):=\left[L^{2}(\Omega), H^{-1}(\Omega)\right]_{s, 2},
$$

and

$$
B^{-s}(\Omega):=\left[L^{2}(\Omega), H^{-1}(\Omega)\right]_{s, \infty} .
$$

Note that, with this notation, $H_{0}^{1 / 2}(\Omega)$ is in fact the space $H_{00}^{1 / 2}(\Omega)$ in the notation of Lions and Magenes [21]. By using (2.7) and (2.8), one can easily check that

$$
\begin{gathered}
\|u\|_{H_{0}^{s}(\Omega)}^{2}=\sum_{k=1}^{\infty} \lambda_{k}^{s}\left\|\left(Q_{k}-Q_{k-1}\right) u\right\|^{2}, \quad \text { for all } u \in H_{0}^{s}(\Omega), \\
\|u\|_{H^{-s}(\Omega)}^{2}=\sum_{k=1}^{\infty} \lambda_{k}^{-s}\left\|\left(Q_{k}-Q_{k-1}\right) u\right\|^{2}, \quad \text { for all } u \in L^{2}(\Omega),
\end{gathered}
$$

and

$$
\|u\|_{B^{-s}(\Omega)}^{2}=\sup _{t>0}\left(t^{2(1-s)} \sum_{k=1}^{\infty} \frac{1}{\lambda_{k}+t^{2}}\left\|\left(Q_{k}-Q_{k-1}\right) u\right\|^{2}\right), \quad \text { for all } u \in L^{2}(\Omega) .
$$

One can verify using the above formula that $B^{0}(\Omega)=L^{2}(\Omega)$ and $B^{-1}(\Omega)=$ $H^{-1}(\Omega)$, which is consistent with the definition (2.5). In addition we have

$$
\sup _{t>0} \frac{t^{2(1-s)}}{\lambda_{k}+t^{2}}=s^{s}(1-s)^{1-s} \lambda_{k}^{-s},
$$


which leads to

$$
\|u\|_{B^{-s}(\Omega)}^{2} \leq s^{s}(1-s)^{1-s}\|u\|_{H^{-s}(\Omega)}^{2} \quad \text { for all } u \in H^{-s}(\Omega),
$$

a well known embedding property.

Lemma 3.1. For $s \in(0,1]$ an equivalent norm on $H_{0}^{s}(\Omega)$ is given by

$$
\|u\|_{s}^{2}:=\|u\|^{2}+\sum_{k=1}^{\infty} \lambda_{k}^{s}\left\|\left(I-Q_{k}\right) u\right\|^{2} .
$$

Proof. Since $H_{0}^{1}(\Omega)$ is dense in $L^{2}(\Omega)$, the union of the spaces $M_{k}$ is also dense in $L^{2}(\Omega)$. Therefore $\lim _{J \rightarrow \infty} Q_{J} u=u$ in $L^{2}(\Omega)$. Thus

$$
\|u\|^{2}=\sum_{k=1}^{\infty}\left\|\left(Q_{k}-Q_{k-1}\right) u\right\|^{2} .
$$

Let $u \in H_{0}^{s}(\Omega)$. Then

$$
\lambda_{J}^{s}\left\|\left(I-Q_{J}\right) u\right\|^{2}=\lambda_{J}^{s} \sum_{k=J+1}^{\infty}\left\|\left(Q_{k}-Q_{k-1}\right) u\right\|^{2} \leq \sum_{k=J+1}^{\infty} \lambda_{k}^{s}\left\|\left(Q_{k}-Q_{k-1}\right) u\right\|^{2} .
$$

Hence, since the the norm on $H_{0}^{s}(\Omega)$ is given by (3.4), given $\epsilon>0$ there exists $J_{0}$ such that, for $J \geq J_{0}, \lambda_{J}^{s}\left\|\left(I-Q_{J}\right) u\right\|^{2}<\epsilon$; that is, $\lim _{J \rightarrow \infty} \lambda_{J}^{s}\left\|\left(I-Q_{J}\right) u\right\|^{2}=0$. On the other hand,

$$
\sum_{k=1}^{J} \lambda_{k}^{s}\left\|\left(Q_{k}-Q_{k-1}\right) u\right\|^{2}=\|u\|^{2}+\left(4^{s}-1\right) \sum_{k=1}^{J-1} \lambda_{k}^{s}\left\|\left(I-Q_{k}\right) u\right\|^{2}-\lambda_{J}^{s}\left\|\left(I-Q_{J}\right) u\right\|^{2},
$$

which proves the lemma.

3.2. Subspace interpolation results. Next, we focus our attention on a specific case of subspace interpolation associated with the pair $X=L^{2}(\Omega)$ and $Y=$ $H^{-1}(\Omega)$. For a fixed $\theta_{0}$ in the interval $(0,1)$, let $\varphi \in L^{2}(\Omega)$ satisfy the following condition:

(C) There exist two positive constants $c_{1}, c_{2}$ such that

$$
c_{1} \lambda_{k}^{-\theta_{0}} \leq\left\|\left(I-Q_{k-1}\right) \varphi\right\|^{2} \leq c_{2} \lambda_{k}^{-\theta_{0}}, k=1,2, \ldots
$$

Lemma 3.2. let $\varphi \in L^{2}(\Omega)$ satisfy $(\mathbf{C})$. Then the following conditions are also satisfied.

(C.0) $\varphi \notin H_{0}^{\theta_{0}}(\Omega)$.

(C.1) There exist two positive constants $c_{1}, c_{2}$ such that

$c_{1} t^{-2 \theta_{0}} \leq(\varphi, \varphi)_{X, t}=\sum_{k=1}^{\infty} \frac{\lambda_{k}}{\lambda_{k}+t^{2}}\left\|\left(Q_{k}-Q_{k-1}\right) \varphi\right\|^{2} \leq c_{2} t^{-2 \theta_{0}}$, for $t>1$.

(C.2) There exist $c_{3}>0$ such that

$$
\left\|\left(Q_{k}-Q_{k-1}\right) \varphi\right\|^{2} \leq c_{3} \lambda_{k}^{-\theta_{0}}, k=1,2, \ldots
$$


Proof. The constants involved in this proof might change at different occurrences. (C.0) follows immediately from Lemma 3.1. Since $\|\varphi\|_{\theta_{0}}$ is not finite, $\varphi \notin H_{0}^{\theta_{0}}(\Omega)$. Using the identity

$$
\left\|\left(Q_{k}-Q_{k-1}\right) u\right\|^{2}=\left\|\left(I-Q_{k-1}\right) u\right\|^{2}-\left\|\left(I-Q_{k}\right) u\right\|^{2}, \quad u \in L^{2}(\Omega),
$$

we have

$$
\begin{aligned}
(\varphi, \varphi)_{X, t} & =\frac{\lambda_{1}\|\varphi\|^{2}}{\lambda_{1}+t^{2}}+t^{2} \sum_{k=1}^{\infty} \frac{\lambda_{k+1}-\lambda_{k}}{\left(\lambda_{k+1}+t^{2}\right)\left(\lambda_{k}+t^{2}\right)}\left\|\left(I-Q_{k}\right) \varphi\right\|^{2} \\
& \geq \frac{3}{4} c_{1} t^{2} \sum_{k=1}^{\infty} \frac{\left(4^{k}\right)^{1-\theta_{0}}}{\left(4^{k}+t^{2}\right)^{2}}=\frac{3}{4} c_{1} t^{-2 \theta_{0}} \sum_{k=1}^{\infty} \frac{\left(4^{k} / t^{2}\right)^{1-\theta_{0}}}{\left(4^{k} / t^{2}+1\right)^{2}} .
\end{aligned}
$$

The last sum can be bounded below by a positive constant independent of $t$ as follows. Let us fix $t \geq 1$ and let $k_{0}$ be the positive integer such that $4^{k_{0}-1} \leq t^{2}<4^{k_{0}}$. Then

$$
\sum_{k=1}^{\infty} \frac{\left(4^{k} / t^{2}\right)^{1-\theta_{0}}}{\left(4^{k} / t^{2}+1\right)^{2}}>\frac{\left(4^{k_{0}} / t^{2}\right)^{1-\theta_{0}}}{\left(4^{k_{0}} / t^{2}+1\right)^{2}} \geq \inf _{x \in[1,4]} \frac{x^{1-\theta_{0}}}{(x+1)^{2}}>0 .
$$

The upper inequality of (C) and (3.8) give

$$
\begin{aligned}
& \sum_{k=1}^{\infty} \frac{\lambda_{k}}{\lambda_{k}+t^{2}}\left\|\left(Q_{k}-Q_{k-1}\right) \varphi\right\|^{2} \\
& \quad \leq c_{2} \sum_{k=1}^{\infty} \frac{\lambda_{k}^{1-\theta_{0}}}{\lambda_{k}+t^{2}} \leq c_{2} t^{-2 \theta_{0}} \sum_{k=1}^{\infty} \frac{\left(4^{k} / t^{2}\right)^{1-\theta_{0}}}{\left(4^{k} / t^{2}+1\right)} \\
& \quad \leq c t^{-2 \theta_{0}} \int_{1}^{\infty} \frac{\left(4^{x} / t^{2}\right)^{1-\theta_{0}}}{\left(4^{x} / t^{2}+1\right)} d x \leq c t^{-2 \theta_{0}} \int_{0}^{\infty} \frac{y^{-\theta_{0}}}{y+1} d y
\end{aligned}
$$

The last inequality is obtained by making the change of variable $4^{x} / t^{2}=y$. Finally, (C.2) follows from (3.8) and the upper inequality of (C).

Lemma 3.3. The space $L^{2}(\Omega)_{\varphi}$ is dense in $H^{-\theta_{0}}(\Omega)$. Thus, the spaces

$$
\left[L^{2}(\Omega)_{\varphi}, H^{-1}(\Omega)\right]_{s, \infty} \quad \text { and } \quad\left[L^{2}(\Omega)_{\varphi}, H^{-1}(\Omega)\right]_{s, 2}
$$

are well defined.

Proof. According to Lemma 2.2 we have to prove that the functional

$$
u \rightarrow(u, \varphi), \quad u \in L^{2}(\Omega),
$$

is not continuous in the topology induced by $H^{-\theta_{0}}(\Omega)$. Let $\left\{u_{n}\right\}$ be the sequence in $L^{2}(\Omega)$ defined by

$$
u_{n}:=\sum_{k=1}^{n} \lambda_{k}^{\theta_{0}} q_{k} \varphi, \text { where } q_{k}=Q_{k}-Q_{k-1}, Q_{0}=0 .
$$

Then,

$$
\left(u_{n}, \varphi\right)=\sum_{k=1}^{n} \lambda_{k}^{\theta_{0}}\left\|q_{k} \varphi\right\|^{2}
$$


On the other hand,

$$
\left\|u_{n}\right\|_{H^{-\theta_{0}}}=\left(\sum_{k=1}^{n} \lambda_{k}^{\theta_{0}}\left\|q_{k} \varphi\right\|^{2}\right)^{1 / 2} .
$$

From (C.0) we have that

$$
\frac{\left(u_{n}, \varphi\right)}{\left\|u_{n}\right\|_{H^{-\theta_{0}}}} \rightarrow \infty \text { as } n \rightarrow \infty .
$$

Therefore, the functional defined in (3.9) is not continuous in the topology induced by $H^{-\theta_{0}}(\Omega)$, and the lemma is proved.

For $u \in L^{2}(\Omega)$ we introduce two other norms, namely

$$
\|u\|_{B_{1}^{-\theta_{0}}}:=\sum_{k=1}^{\infty} \lambda_{k}^{-\theta_{0} / 2}\left\|\left(Q_{k}-Q_{k-1}\right) u\right\|
$$

and

$$
\|u\|_{B_{01}^{-\theta_{0}}}^{2}:=\|u\|_{B^{-\theta_{0}}}^{2}+\|u\|_{B_{1}^{-\theta_{0}}}^{2} .
$$

The space $B_{01}^{-\theta_{0}}$ is defined as completion of $L^{2}(\Omega)$ with respect to the norm $\|\cdot\|_{B_{01}^{-\theta_{0}}}$.

Theorem 3.1. Let $\varphi \in L^{2}(\Omega)$ satisfy $(\boldsymbol{C})$. Then, there exists a positive constant c such that

$$
\|u\|_{\left[L^{2}(\Omega)_{\varphi}, H^{-1}(\Omega)\right]_{\theta_{0}, \infty}} \leq c\|u\|_{B_{01}^{-\theta_{0}}} \quad \text { for all } u \in B_{01}^{-\theta_{0}} .
$$

Proof. Recall that $L^{2}(\Omega)=X$ and $H^{-1}(\Omega)=Y$. Since $L^{2}(\Omega)$ is dense in $B_{01}^{-\theta_{0}}$, it is enough to prove (3.11) on $L^{2}(\Omega)$. Let us further note here that by proving (3.11) on $L^{2}(\Omega)$ we have in particular the inclusion $L^{2}(\Omega) \subset\left[L^{2}(\Omega)_{\varphi}, H^{-1}(\Omega)\right]_{\theta_{0}, \infty}$.

For $u \in X$ and $w_{\varphi}$ as defined in the proof of Theorem 2.1.

$$
\begin{aligned}
\left(w_{\varphi}, u\right)_{Y}=\left(\left(I+t^{2} S_{\varphi}^{-1}\right)^{-1} u, u\right)_{Y} & =(u, u)_{Y}-t^{2}\left(S_{\varphi}^{-1}\left(I+t^{2} S_{\varphi}^{-1}\right)^{-1} u, u\right)_{Y} \\
& \leq(u, u)_{Y} \leq c\left(\theta_{0}\right)\|u\|_{B^{-\theta_{0}}}^{2} .
\end{aligned}
$$

Then, using (2.20), (2.24) and the above estimate, we have that

$$
\begin{aligned}
\|u\|_{\left[X_{\varphi}, Y\right]_{\theta_{0}, \infty}}^{2} & \leq \sup _{0<t \leq 1} t^{2\left(1-\theta_{0}\right)}\left(w_{\varphi}, u\right)_{Y}+\sup _{t>1} t^{2\left(1-\theta_{0}\right)}\left(w_{\varphi}, u\right)_{Y} \\
& \leq c\left(\theta_{0}\right) \sup _{0<t \leq 1} t^{2\left(1-\theta_{0}\right)}\|u\|_{B^{-\theta_{0}}}^{2}+\sup _{t>1} t^{2\left(1-\theta_{0}\right)}\left(w_{\varphi}, u\right)_{Y} \\
& \leq c\left(\theta_{0}\right)\|u\|_{B^{-\theta_{0}}}^{2}+\sup _{t>1} t^{2\left(1-\theta_{0}\right)}(w, u)_{Y}+\sup _{t>1} t^{4-2 \theta_{0}} \frac{\left|(u, \varphi)_{Y, t}\right|^{2}}{(\varphi, \varphi)_{X, t}} .
\end{aligned}
$$

Note that

$$
\sup _{t>1} t^{2\left(1-\theta_{0}\right)}(w, u)_{Y} \leq \sup _{t>0} t^{2\left(1-\theta_{0}\right)}(w, u)_{Y}=\|u\|_{B^{-\theta_{0}}}^{2} .
$$

Hence, the inequality (3.11) is satisfied if one can find a positive constant $c=$ $c\left(\theta_{0}, c_{1}, c_{2}\right)$ such that

$$
\sup _{t>1} t^{4-2 \theta_{0}} \frac{\left|(u, \varphi)_{Y, t}\right|^{2}}{(\varphi, \varphi)_{X, t}} \leq c\|u\|_{B_{1}^{-\theta_{0}}}^{2}, \quad \text { for all } u \in X .
$$

In the view of condition (C.1) this reduces to

$$
\sup _{t>1} t^{4}\left|(u, \varphi)_{Y, t}\right|^{2} \leq c\|u\|_{B_{1}^{-\theta_{0}}}^{2}, \quad \text { for all } u \in X .
$$


Here $(\cdot, \cdot)_{X}$ and $\|\cdot\|_{X}$ are simply the $L^{2}(\Omega)$ inner product $(\cdot, \cdot)$ and the $L^{2}(\Omega)$ norm $\|\cdot\|$. We denote $Q_{k}-Q_{k-1}$ by $q_{k}$, with $Q_{0}=0$.

For $u \in X$, we have

$$
(u, \varphi)_{Y, t}=\sum_{k=1}^{\infty} \frac{1}{\lambda_{k}+t^{2}}\left(q_{k} u, q_{k} \varphi\right) .
$$

By using condition (C.2), we obtain the estimate

$$
\left|(u, \varphi)_{Y, t}\right| \leq c_{2} \sum_{k=1}^{\infty} \frac{\lambda_{k}^{-\theta_{0} / 2}}{\lambda_{k}+t^{2}}\left\|q_{k} u\right\|
$$

The function

$$
t \rightarrow t^{2} \sum_{k=1}^{\infty} \frac{\lambda_{k}^{-\theta_{0} / 2}}{\lambda_{k}+t^{2}}\left\|q_{k} u\right\|
$$

is an increasing function of $t$. As $t \rightarrow \infty$, the limit of the above function is exactly $\|u\|_{B_{1}^{-\theta_{0}}}$. Therefore (13.13) holds, and the proof is complete.

Following the proof of the above theorem (and using the upper part of (C.2)), we can characterize the norm of the intermediate space $\left[L^{2}(\Omega)_{\varphi}, H^{-1}(\Omega)\right]_{\theta_{0}, \infty}$.

Corollary 3.1. An equivalent norm on the space $\left[L^{2}(\Omega)_{\varphi}, H^{-1}(\Omega)\right]_{\theta_{0}, \infty}$ is given by

$$
\sup _{t>1} t^{2}\left|(u, \varphi)_{Y, t}\right|+\|u\|_{B^{-\theta_{0}}}=\sup _{x>1}\left|\sum_{k=1}^{\infty} \frac{x}{\lambda_{k}+x}\left(q_{k} u, \varphi\right)\right|+\|u\|_{B^{-\theta_{0}}} .
$$

Remark 3.1. It is not difficult to see that the embedding stated in Theorem 3.1 still holds if we replace $\|u\|_{B_{1}^{-\theta_{0}}}$ in the definition of $\|u\|_{B_{01}^{-\theta_{0}}}$ with the following semi-norm:

$$
|u|_{\varphi}:=\sum_{k=1}^{\infty}\left|\left(q_{k} u, \varphi\right)\right| .
$$

In either case one can immediately check that

$$
|u|_{\varphi} \leq\|u\|_{B_{1}^{-\theta_{0}}} \leq\|u\|_{B_{01}^{-\theta_{0}}} \leq c\|u\|_{H_{a}^{-\theta_{0}}}, \quad \text { for all } u \in L^{2}(\Omega),
$$

where $a=\left\{a_{k}\right\}_{k \geq 1}$ is any sequence of positive real numbers such that

$$
\sum_{k=1}^{\infty} \frac{1}{a_{k}^{2}}<\infty
$$

and

$$
\|u\|_{H_{a}^{-\theta_{0}}}^{2}:=\sum_{k=1}^{\infty} \lambda_{k}^{-\theta_{0}} a_{k}^{2}\left\|q_{k} u\right\|^{2}, u \in L^{2}(\Omega)
$$

For example, we can take $a_{k}=k, a_{k}=k^{1 / 2} \ln (k+1)$ or $\lambda_{k}^{\epsilon / 2}$. In particular, taking $a_{k}=\lambda_{k}^{\epsilon / 2}$, with $\epsilon \leq 1+\theta_{0}$, we have

$$
\|u\|_{B_{01}^{-\theta_{0}}} \leq c \epsilon^{-1 / 2}\|u\|_{H^{-\theta_{0}+\epsilon}} \quad \text { for all } u \in H^{-\theta_{0}+\epsilon},
$$

where $c$ is a constant independent of $\epsilon$. 
One consequence of the above remark is that the norm $\left[L^{2}(\Omega)_{\varphi}, H^{-1}(\Omega)\right]_{\theta_{0}, \infty}$ in (3.11) can be replaced by $\|\cdot\|_{H^{-\theta_{0}+\epsilon}}$.

It is known (see [2]) that if (C.0)-(C.2) are satisfied, for $\theta_{0}<\theta \leq 1$, then

$$
\left[L^{2}(\Omega)_{\varphi}, H^{-1}(\Omega)\right]_{\theta, 2}=\left[L^{2}(\Omega), H^{-1}(\Omega)\right]_{\theta, 2}:=H^{-\theta}(\Omega) .
$$

Using Lemma 3.3. we see that $L^{2}(\Omega)_{\varphi}$ is dense in $H^{-\theta}(\Omega)$ if and only if $\theta_{0} \leq \theta \leq 1$. Thus one might expect that (3.16) holds for the critical value $\theta=\theta_{0}$. A weaker result, but in some sense a better result, compared with Theorem [3.1 would be: There exists a positive constant $c$ such that

$$
\|u\|_{\left[L^{2}(\Omega) \varphi, H^{-1}(\Omega)\right]_{\theta_{0}, \infty}} \leq c\|u\|_{H^{-\theta_{0}(\Omega)}} \quad \text { for all } u \in H^{-\theta_{0}}(\Omega) .
$$

The next theorem proves that this is not true, and consequently that (3.16) cannot hold for the critical value $\theta=\theta_{0}$. In addition, Theorem 3.1 cannot be "essentially" improved.

Theorem 3.2. The space $H^{-\theta_{0}}(\Omega)$ is not continuously embedded in

$$
\left[L^{2}(\Omega)_{\varphi}, H^{-1}(\Omega)\right]_{\theta_{0}, \infty} .
$$

Proof. Consider the sequence $\left\{u_{n}\right\}$ defined by

$$
u_{n}:=\sum_{k=1}^{n} \frac{\lambda_{k}^{\theta_{0}}}{k} q_{k} \varphi \in L^{2}(\Omega) \text {. }
$$

First, note that $u_{n}$ is in both $H^{-\theta_{0}}(\Omega)$ and $\left[L^{2}(\Omega)_{\varphi}, H^{-1}(\Omega)\right]_{\theta_{0}, \infty}$. We have $q_{k} u_{n}=$ $\frac{\lambda_{k}^{\theta_{0}}}{k} q_{k} \varphi$, for $k=1, \ldots, n$, and $q_{k} u_{n}=0$, for $k>n$. By (C.2), we see that the sequence

$$
\left\|u_{n}\right\|_{H^{-\theta_{0}}}^{2}=\sum_{k=1}^{n} \frac{\lambda_{k}^{\theta_{0}}}{k^{2}}\left\|q_{k} \varphi\right\|^{2}, n=1,2, \ldots
$$

is bounded. Let $k_{0}$ be the smallest nonnegative integer so that $\frac{4^{\theta_{0}}}{k_{0}+1}-\frac{1}{k_{0}}>0$, and choose $n$ such that $n>n_{0}$. According to Corollary 3.1 and (C), for a positive constant $c$

$$
\begin{aligned}
c\left\|u_{n}\right\|_{\left[L^{2}(\Omega)_{\varphi}, H^{-1}(\Omega)\right]_{\theta_{0}, \infty}} \geq \sup _{x>1}\left|\sum_{k=1}^{\infty} \frac{x}{\lambda_{k}+x}\left(q_{k} u_{n}, \varphi\right)\right| \\
=\sup _{x>1} \sum_{k=1}^{n}\left(\frac{x}{\lambda_{k}+x}\right) \frac{\lambda_{k}^{\theta_{0}}}{k}\left\|q_{k} \varphi\right\|^{2}=\sum_{k=1}^{n} \frac{\lambda_{k}^{\theta_{0}}}{k}\left\|q_{k} \varphi\right\|^{2} \\
=\sum_{k=1}^{n} \frac{\lambda_{k}^{\theta_{0}}}{k}\left(\left\|\left(I-Q_{k-1}\right) \varphi\right\|^{2}-\left\|\left(I-Q_{k}\right) \varphi\right\|^{2}\right) \\
\geq \sum_{k=1}^{n-1}\left\|\left(I-Q_{k}\right) \varphi\right\|^{2}\left(\frac{\lambda_{k+1}^{\theta_{0}}}{k+1}-\frac{\lambda_{k}^{\theta_{0}}}{k}\right)-\frac{\lambda_{n}^{\theta_{0}}}{n}\left\|\left(I-Q_{n}\right) \varphi\right\|^{2} \\
\geq \frac{c_{1}}{4^{\theta_{0}}} \sum_{k=k_{0}}^{n-1}\left(\frac{4^{\theta_{0}}}{k+1}-\frac{1}{k}\right)+\frac{c_{2}}{4^{\theta_{0}}} \sum_{k=1}^{k_{0}-1}\left(\frac{4^{\theta_{0}}}{k+1}-\frac{1}{k}\right)-\frac{c_{2}}{n},
\end{aligned}
$$

which proves that $\left\|u_{n}\right\|_{\left[L^{2}(\Omega)_{\varphi}, H^{-1}(\Omega)\right]_{\theta_{0}, \infty}} \rightarrow \infty$ as $n \rightarrow \infty$, and contradicts (3.17). 


\section{Applications to Shift theorem \\ FOR THE LAPLACE OPERATOR ON POLYGONAL DOMAINS}

In this section we will prove that the estimate (1.2) holds for the case $s=s_{0}$ provided the fractional Sobolev norms in (1.2) are appropriately replaced.

4.1. Shift estimate for the critical value. Let $\Omega$ be a nonconvex polygonal domain in $\mathbb{R}^{2}$, with boundary $\partial \Omega$, and with only one re-entrant corner of measure $\omega$ and let $s_{0}=\pi / \omega$. In the general case when $\partial \Omega$ has more than one re-entrant corner the result of this section can be reduced by a partition of unity type argument to this case. Given $f \in L^{2}(\Omega)$, we consider the Dirichlet problem (1.1). The variational formulation of (1.1) is: Find $u \in H_{0}^{1}(\Omega)$ such that

$$
\int_{\Omega} \nabla u \cdot \nabla v d x=\int_{\Omega} f v d x \quad \text { for all } v \in H_{0}^{1}(\Omega) .
$$

It is well known that for $f \in L^{2}(\Omega)$ the variational problem has a unique solution $u \in H_{0}^{1}(\Omega)$, and

$$
\|u\|_{H^{1}(\Omega)} \leq c\|f\|_{H^{-1}(\Omega)} \quad \text { for all } f \in L^{2}(\Omega),
$$

where $H^{-1}(\Omega)$ is the dual of $H_{0}^{1}(\Omega)$. By taking $v$ in $\mathcal{D}(\Omega)$, the space of all infinitely differentiable functions with compact support in $\Omega$, one has that the solution $u$ of (4.1) satisfies $-\Delta u=f$ in the sense of distributions in $\Omega$, so the equality is satisfied pointwise, almost everywhere in $\Omega$. Also, since $u$ belongs to $H_{0}^{1}(\Omega)$ the boundary conditions of (1.1) is automatically fulfilled.

Let $V^{2}(\Omega):=H^{2}(\Omega) \cap H_{0}^{1}(\Omega)$. Then (see, e.g., Theorem 2.2.3 in [16]) the Laplace operator $\Delta: V^{2}(\Omega) \rightarrow L^{2}(\Omega)$ is a Fredholm operator. In fact we have that

$$
\|u\|_{H^{2}(\Omega)} \leq c\|\Delta u\|_{L^{2}(\Omega)} \quad \text { for all } u \in V^{2}(\Omega),
$$

and the range of the operator has codimension one. Grisvard characterized the orthogonal complement $\mathcal{N}$ of the range of the Laplace operator for the case of a polygonal domain (see [15], 16]). If $\zeta \in \mathcal{D}(\Omega)$ is a cut-off function, which depends only on the distance $r$ to the re-entrant corner, is identically equal to one in a neighborhood of the re-entrant corner and is identically equal to zero close to the part of $\partial \Omega$ which does not contain the sides of the re-entrant corner, then $\mathcal{N}=$ $\operatorname{span}\{\psi\}$, where $\psi=\varphi+u^{R}, \varphi(r, \theta)=\zeta r^{-s_{0}} \sin \left(s_{0} \theta\right)$ and $u^{R} \in H_{0}^{1}(\Omega)$ is the variatonal solution of 1.1 with $f=\Delta \varphi$.

If we define $T: H^{-1}(\Omega) \rightarrow H_{0}^{1}(\Omega)$ by $T f:=u$, where $u$ is the solution of (4.1), then $T$ is a bounded operator. Moreover, $T$ is a bounded operator from $L^{2}(\Omega)_{\psi}$ to $H^{2}(\Omega) \cap H_{0}^{1}(\Omega)$. Thus, by interpolation, we have

$$
\|u\|_{\left[H^{2}(\Omega) \cap H_{0}^{1}(\Omega), H_{0}^{1}(\Omega)\right]_{1-s_{0}, \infty}} \leq c\|f\|_{\left[L^{2}(\Omega)_{\psi}, H^{-1}(\Omega)\right]_{1-s_{0}, \infty}}
$$

for all $f \in\left[L^{2}(\Omega)_{\psi}, H^{-1}(\Omega)\right]_{1-s_{0}, \infty}$.

Let $B^{1+s_{0}}(\Omega)$ denote the Besov space $\left[H^{2}(\Omega), H^{1}(\Omega)\right]_{1-s_{0}, \infty}$. Then, we have the following theorem.

Theorem 4.1. Let $u$ be the variational solution of (1.1). Then there exist positive constant $c_{1}$ and $c_{2}$ such that

$$
\left\{\begin{aligned}
\|u\|_{B^{1+s_{0}(\Omega)}} & \leq\|u\|_{\left[H^{2} \cap H_{0}^{1}, H_{0}^{1}\right]_{1-s_{0}, \infty}} \leq c_{1}\|f\|_{\left[L_{\psi}^{2}, H^{-1}\right]_{1-s_{0}, \infty}} \\
& \leq c_{2}\|f\|_{B_{01}^{-1+s_{0}}(\Omega)}, \quad \text { for all } f \in B_{01}^{-1+s_{0}}(\Omega) .
\end{aligned}\right.
$$


Proof. Use (4.4) and apply Theorem 3.1 with $\theta_{0}=1-s_{0}$ and $\varphi=\psi$. The proof that $(\mathbf{C})$ is satisfied is given later. The lower part of (4.5) follows by comparing the $K$ functions associated with the two intermediate spaces.

4.2. Proving condition (C). Let $\Omega$ be a polygonal-sector domain (Figure 1 ).

$$
\bar{\Omega}=\bigcup_{i=1}^{n} \overline{\tau_{i}}
$$

where, for $i=1, \ldots, n, \tau_{i}$ is a triangular domain with vertices $S_{i}, O, S_{i+1}$, and $O$ is taken to be the origin of a Cartesian system of coordinates in the plane.

For $i=1, \ldots, n+1$, let $\Gamma_{i}$ denote the segment $\left[O, S_{i}\right]$. We assume, without loss of generality, that $S_{1}$ lies on the positive semi-axis and the length of $\left[O, S_{i}\right]$ is not greater than one.

Let $\mathcal{T}_{1}=\left\{\tau_{1}, \ldots, \tau_{n}\right\}$ be the initial triangulation of $\Omega$. We define multilevel triangulations recursively. For $k>1$, the triangulation $\mathcal{T}_{k}$ is obtained from $\mathcal{T}_{k-1}$ by splitting each triangle in $\mathcal{T}_{k-1}$ into four triangles by connecting the midpoints of the edges. The space $M_{k}$ is defined to be the space of all functions which are piecewise linear with respect to $\mathcal{T}_{k}$, vanish on $\partial \Omega$ and are continuous on $\Omega$. Let $Q_{k}$ denote the $L^{2}(\Omega)$ orthogonal projection onto $M_{k}$.

Let $\psi=\varphi+u^{R}$, where $\varphi(r, \theta)=\zeta r^{-s_{0}} \sin \left(s_{0} \theta\right)$ and $u^{R} \in H_{0}^{1}(\Omega)$. We verify that the function $\psi$ satisfies the condition (C) with $\theta_{0}=1-s_{0}$.

To begin with, we will prove that the function $\varphi$ satisfies (C). Let $\bar{M}_{k}$ be the space of piecewise linear (possibly discontinuous) functions with respect to $\mathcal{T}_{k}$ defined on $\Omega$, and let $\bar{Q}_{k}$ be the $L^{2}(\Omega)$ orthogonal projection onto $\bar{M}_{k}$. The first step is to prove that there exists a positive constant $c$ such that

$$
\left\|\left(I-\bar{Q}_{k}\right) \varphi\right\|^{2} \geq c \lambda_{k}^{-\theta_{0}}, k=1,2, \ldots
$$

We define $\tau_{1}^{k}$ to be the triangle in $\mathcal{T}_{k}$ which is the image of $\tau_{1} \in \mathcal{T}_{1}$ via the map $\hat{x} \rightarrow h_{k} \hat{x}$. Here, without loss of generality, we assume that $h_{k}^{2}=\lambda_{k}^{-1}=4^{-k+1}$. Then

$$
\left\|\left(I-\bar{Q}_{k}\right) \varphi\right\|_{L^{2}(\Omega)}^{2} \geq\left\|\left(I-\bar{Q}_{k}\right) \varphi\right\|_{L^{2}\left(\tau_{1}^{k}\right)}^{2}=\|\varphi\|_{L^{2}\left(\tau_{1}^{k}\right)}^{2}-\left\|\bar{Q}_{k} \varphi\right\|_{L^{2}\left(\tau_{1}^{k}\right)}^{2} .
$$

The projection $\bar{Q}_{k} \varphi$ can be estimated on $\tau_{1}^{k}$ in terms of the three nodal functions $\varphi_{1}^{k}, \varphi_{2}^{k}, \varphi_{3}^{k}$ associated with the three vertices of $\tau_{1}^{k}$. If $M^{k}$ is the $3 \times 3$ Gram matrix

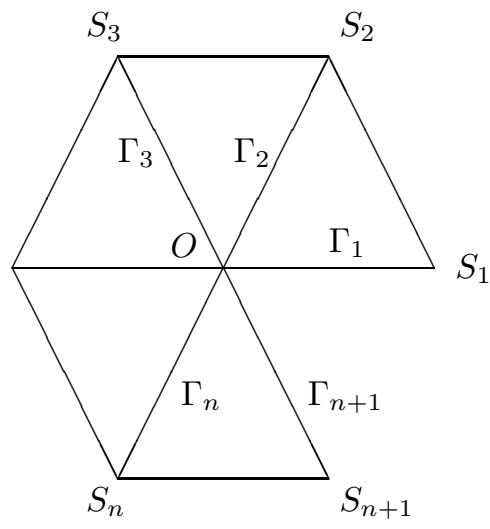

Figure 1. Polygonal domain 
associated with the set $\left\{\varphi_{1}^{k}, \varphi_{2}^{k}, \varphi_{3}^{k}\right\}$, and $S^{k}:=\left(S_{i j}^{k}\right), i, j=1,2,3$, is the inverse of $M^{k}$, then

$$
\|\varphi\|_{L^{2}\left(\tau_{1}^{k}\right)}^{2}-\left\|\bar{Q}_{k} \varphi\right\|_{L^{2}\left(\tau_{1}^{k}\right)}^{2}=\int_{\tau_{1}^{k}} \varphi^{2} d x-\sum_{i, j=1}^{3} S_{i j}^{k} \int_{\tau_{1}^{k}} \varphi \varphi_{i}^{k} d x \int_{\tau_{1}^{k}} \varphi \varphi_{j}^{k} d x .
$$

Further, by making the change of variable $x=h_{k} \hat{x}$ in the above integrals, a simple computation shows that

$$
\begin{aligned}
\|\varphi\|_{L^{2}\left(\tau_{1}^{k}\right)}^{2}-\left\|\bar{Q}_{k} \varphi\right\|_{L^{2}\left(\tau_{1}^{k}\right)}^{2} & =h_{k}^{2-2 s_{0}}\left(\int_{\tau_{1}} \varphi^{2} d \hat{x}-\sum_{i, j=1}^{3} S_{i j}^{1} \int_{\tau_{1}} \varphi \varphi_{i}^{1} d \hat{x} \int_{\tau_{1}} \varphi \varphi_{j}^{1} d \hat{x}\right) \\
& =\lambda_{k}^{-\theta_{0}}\left(\|\varphi\|_{L^{2}\left(\tau_{1}\right)}^{2}-\left\|\bar{Q}_{1} \varphi\right\|_{L^{2}\left(\tau_{1}\right)}^{2}\right) .
\end{aligned}
$$

Since $\varphi$ is not linear on $\tau_{1}$, the constant $\|\varphi\|_{L^{2}\left(\tau_{1}\right)}^{2}-\left\|\bar{Q}_{1} \varphi\right\|_{L^{2}\left(\tau_{1}\right)}^{2}$ is strictly positive. Combining the above estimates, we have proven that (4.6) holds.

The second step is to use (4.6) and the fact that $M_{k}$ is a subspace of $\bar{M}_{k}$, in order to obtain

$$
\left\|\left(I-Q_{k}\right) \varphi\right\|^{2} \geq c \lambda_{k}^{-\theta_{0}}, k=1,2, \ldots
$$

Next, we prove that there exists a positive constant $c$ such that

$$
\left\|\left(I-Q_{k}\right) \varphi\right\|^{2} \leq c \lambda_{k}^{-\theta_{0}}, k=1,2, \ldots
$$

Let $\eta_{k}$ be a cutoff function which depends only on $r$ and satisfies

$$
\eta_{k}(r)=0 \text { for } r \leq h_{k}, \eta_{k}(r)=1 \text { for } r \geq 2 h_{k},
$$

$$
\left|\eta_{k}^{\prime}(r)\right| \leq c / h_{k}, \quad\left|\eta_{k}^{\prime \prime}(r)\right| \leq c / h_{k}^{2} \quad \text { for all } h_{k} \leq r \leq 2 h_{k}, k=1,2, \ldots,
$$

for some positive constant $c$. For example, we can take

$$
\eta_{k}(r)=\frac{1}{2}+\frac{1}{2} \sin \left(\frac{\pi r}{h_{k}}-\frac{3 \pi}{2}\right) \text { on }\left[h_{k}, 2 h_{k}\right] .
$$

Then, $\varphi=\left(1-\eta_{k}\right) \varphi+\eta_{k} \varphi$ and $\eta_{k} \varphi \in H^{2}(\Omega)$. Let $\Pi_{k}: H^{2}(\Omega) \rightarrow M_{k}$ be the interpolant associated with $\mathcal{T}_{k}$. By applying standard approximation properties and (4.3) we obtain

$$
\begin{aligned}
\left\|\left(I-Q_{k}\right) \varphi\right\| & \leq\left\|\left(I-Q_{k}\right)\left(1-\eta_{k}\right) \varphi\right\|+\left\|\left(I-Q_{k}\right) \eta_{k} \varphi\right\| \\
& \leq\left\|\left(1-\eta_{k}\right) \varphi\right\|+\left\|\left(I-\Pi_{k}\right) \eta_{k} \varphi\right\| \\
& \leq\left\|\left(1-\eta_{k}\right) \varphi\right\|+c h_{k}^{2}\left\|\eta_{k} \varphi\right\|_{H^{2}(\Omega)} \\
& \leq\left\|\left(1-\eta_{k}\right) \varphi\right\|+c h_{k}^{2}\left\|\Delta\left(\eta_{k} \varphi\right)\right\|_{L^{2}(\Omega)} .
\end{aligned}
$$

Using a simple computation in polar coordinates, and the estimates for the derivative of $\eta_{k}$, we get

$$
\left\|\left(1-\eta_{k}\right) \varphi\right\|^{2} \leq \operatorname{ch}_{k}^{2 \theta_{0}} \quad \text { for all } k=1,2, \ldots
$$

and

$$
h_{k}^{2}\left\|\Delta\left(\eta_{k} \varphi\right)\right\|_{L^{2}(\Omega)} \leq c h_{k}^{2 \theta_{0}} \quad \text { for all } k=1,2, \ldots
$$

Combining the above inequalities, we conclude that (4.8) is valid. Thus, (C) holds for the function $\varphi$. Since the function $u_{R}$ belongs to $H_{0}^{1}(\Omega)$, we have

$$
\left\|\left(I-Q_{k}\right) u_{R}\right\|^{2} \leq c \lambda_{k}^{-1}, k=1,2, \ldots .
$$

Therefore, the function $\psi$ satisfies condition (C). 


\section{Applications to finite element Convergence estimates}

Let $\Omega$ be a nonconvex polygonal domain in $\mathbb{R}^{2}$, with boundary $\partial \Omega$, and with only one re-entrant corner of measure $\omega$, and let $s_{0}=\pi / \omega, \theta_{0}=1-s_{0}$. Let $u \in H_{0}^{1}(\Omega)$ be the variational solution of (4.1) with $f \in B_{01}^{-1+s_{0}}$, and let $V_{h}$ be a finite dimensional approximation subspace of $H_{0}^{1}(\Omega)$. We consider the discrete problem:

Find $u_{h} \in V_{h}$ such that

$$
\int_{\Omega} \nabla u_{h} \cdot \nabla v d x=\int_{\Omega} f v d x \quad \text { for all } v \in V_{h}
$$

Further, let us assume that

$$
\left\|u-u_{h}\right\|_{H^{1}(\Omega)} \leq c\|u\|_{H^{1}(\Omega)}, \quad \text { for all } u \in H_{0}^{1}(\Omega),
$$

and

$$
\left\|u-u_{h}\right\|_{H^{1}(\Omega)} \leq \operatorname{ch}\|u\|_{H^{2}(\Omega)}, \quad \text { for all } u \in H^{2}(\Omega) \cap H_{0}^{1}(\Omega) .
$$

By interpolation with $p=\infty$ and $s=1-s_{0}$, from (5.2) and (5.3), we obtain that

$$
\left\|u-u_{h}\right\|_{H^{1}(\Omega)} \leq c h^{s_{0}}\|u\|_{\left[H^{2}(\Omega) \cap H_{0}^{1}(\Omega), H_{0}^{1}(\Omega)\right]_{1-s_{0}, \infty}},
$$

for all $u \in\left[H^{2}(\Omega) \cap H_{0}^{1}(\Omega), H_{0}^{1}(\Omega)\right]_{1-s_{0}, \infty}$, where $c$ is a constant independent of $h$.

We thus have the following result.

Theorem 5.1. Let $u, u_{h}$ be the variational solutions of problems (4.1) and (5.1), respectively. Then, there exists a constant $c$ independent of $h$ such that

$$
\left\|u-u_{h}\right\|_{H^{1}(\Omega)} \leq c h^{s_{0}}\|f\|_{B_{01}^{-1+s_{0}}(\Omega)}, \quad \text { for all } f \in B_{01}^{-1+s_{0}}(\Omega) .
$$

Consequently, for $s_{0}<s \leq 1+s_{0}$ there exists a constant $c$, independent of $h$ and s, such that

$$
\left\|u-u_{h}\right\|_{H^{1}(\Omega)} \leq c\left(s-s_{0}\right)^{-1 / 2} h^{s_{0}}\|f\|_{H^{-1+s}(\Omega)}, \quad \text { for all } f \in H^{-1+s}(\Omega) .
$$

and

$$
\left\|u-u_{h}\right\|_{H^{1-s}(\Omega)} \leq c\left(s-s_{0}\right)^{-1} h^{2 s_{0}}\|f\|_{H^{-1+s}(\Omega)}, \quad \text { for all } f \in H^{-1+s}(\Omega) .
$$

Proof. Inequality (5.5) follows from (5.4) and Theorem 4.1. By (3.15) with $\theta_{0}=$ $1-s_{0}$ and $\epsilon=s-s_{0}$, (5.6) follows from (5.5). Inequality (5.7) is obtained using a standard duality argument.

Let us note that according to Theorem 4.1 the estimate (5.5) holds for $f$ in the space $\left[L^{2}(\Omega)_{\psi}, H^{-1}(\Omega)\right]_{1-s_{0}, \infty}$, where $\psi$ is the function defined in the previous section.

It was proved in [14] that $\left\|u-u_{h}\right\|_{H^{1}(\Omega)} \geq c h^{s_{0}}$, and $\left\|u-u_{h}\right\|_{L^{2}(\Omega)} \geq c h^{2 s_{0}}$, unless $u \in H^{1+s_{0}}(\Omega)$. Further, in [26], for $s \geq 1$, it is shown that $\left\|u-u_{h}\right\|_{H^{1-s}(\Omega)} \geq$ $c h^{2 s_{0}}$, under the same condition. In general, we know that $u \notin H^{1+s_{0}}(\Omega)$ even if $f$ is regular. For example, this is the case if $f$ is in $L^{2}(\Omega)$ but not in $L^{2}(\Omega)_{\psi}$. Using Theorem 5.1, this means that

$$
\left\|u-u_{h}\right\|_{H^{1}(\Omega)} \approx c h^{s_{0}}
$$

and, for $s>s_{0}$,

$$
\left\|u-u_{h}\right\|_{H^{1-s}(\Omega)} \approx c h^{2 s_{0}}
$$


In view of Theorem [3.2, (5.6) does not hold with $s=s_{0}$. Nevertheless, by choosing an appropriate $s$ (as a function of $h$ ) we can use (5.6) to deduce a similar estimate for $f \in H^{-1+s_{0}}(\Omega)$.

Theorem 5.2. Let $u, u_{h}$ be the variational solutions of problems (4.1) and (5.1), respectively. Then, there exists a constant $c$, independent of $h$, with $h \leq e^{-1 / 2}$, such that

$$
\left\|u-u_{h}\right\|_{H^{1}(\Omega)} \leq c h^{s_{0}}(\log 1 / h)^{1 / 2}\|f\|_{H^{-1+s_{0}}(\Omega)} \quad \text { for all } f \in H^{-1+s_{0}}(\Omega)
$$

and

$$
\left\|u-u_{h}\right\|_{H^{1-s_{0}}(\Omega)} \leq c h^{2 s_{0}}(\log 1 / h)\|f\|_{H^{-1+s_{0}}(\Omega)} \quad \text { for all } f \in H^{-1+s_{0}}(\Omega) .
$$

Proof. Let $f \in H^{-1+s_{0}}(\Omega)$ and write $f=f-Q_{h} f+Q_{h} f$. Next, we denote by $u^{h}$ the solution of

$$
\int_{\Omega} \nabla u^{h} \cdot \nabla v d x=\int_{\Omega} Q_{h} f v d x \quad \text { for all } v \in H_{0}^{1}(\Omega) .
$$

Since $u-u^{h}$ is the solution of (4.1) with $f-Q_{h} f$ instead of $f$, we have

$$
\left\|u-u^{h}\right\|_{H^{1}(\Omega)} \leq c\left\|f-Q_{h} f\right\|_{H^{-1}(\Omega)},
$$

and from standard approximation properties we get

$$
\left\|f-Q_{h} f\right\|_{H^{-1}(\Omega)} \leq c h^{s_{0}}\|f\|_{H^{-1+s_{0}(\Omega)}}
$$

Combining the two inequalities, we have

$$
\left\|u-u^{h}\right\|_{H^{1}(\Omega)} \leq c h^{s_{0}}\|f\|_{H^{-1+s_{0}(\Omega)}} .
$$

Next, using the estimate (5.6) of Theorem[5.1, a negative norms inverse inequality and the stability of the $L^{2}$ projection in $H^{-1+s_{0}}(\Omega)$, we get

$$
\begin{aligned}
\left\|u^{h}-u_{h}\right\|_{H^{1}(\Omega)} & \leq c\left(s-s_{0}\right)^{-1 / 2} h^{s_{0}}\left\|Q_{h} f\right\|_{H^{-1+s}(\Omega)} \\
& \leq c h^{s_{0}}\left(s-s_{0}\right)^{-1 / 2} h^{s_{0}-s}\left\|Q_{h} f\right\|_{H^{-1+s_{0}}(\Omega)} \\
& \leq c h^{s_{0}}\left(s-s_{0}\right)^{-1 / 2} h^{s_{0}-s}\|f\|_{H^{-1+s_{0}}(\Omega)}
\end{aligned}
$$

where $c$ is a constant independent of $h$ and $s$. The minimum of the function $s \rightarrow\left(s-s_{0}\right)^{-1 / 2} h^{s_{0}-s}$ on the interval $\left(s_{0}, 1+s_{0}\right]$ is $(2 e \log 1 / h)^{1 / 2}$ and is attained for $s=s_{0}+(2 \log 1 / h)^{-1}$, with $h \leq e^{-1 / 2}$. Thus,

$$
\left\|u^{h}-u_{h}\right\|_{H^{1}(\Omega)} \leq c h^{s_{0}}(\log 1 / h)^{1 / 2}\|f\|_{H^{-1+s_{0}(\Omega)}} .
$$

Finally, (5.8) follows from (5.10) and (5.11).

The estimate (5.9) follows from a duality argument. 


\section{REFERENCES}

1. R. A. Adams. Sobolev Spaces. Academic Press, New York, 1975. MR 56:9247

2. C. Bacuta, J. H. Bramble, J. Pasciak. New interpolation results and applications to finite element methods for elliptic boundary value problems. East-West J. Numer. Math. 9:179-198, 2001. MR 2002h:41053

3. C. Bennett and R. Sharpley. Interpolation of Operators. Academic Press, New-York, 1988. MR 89e:46001

4. J. Bergh, J. Löström. Interpolation Spaces. An Introduction, Springer-Verlag, New York, 1976. MR 58:2349

5. D. Braess. Finite Elements. Theory, Fast Solvers, and Applications in Solid Mechanics. Cambridge University Press, Cambridge, 1997. MR 98f:65002

6. J. H. Bramble. Interpolation between Sobolev spaces in Lipschitz domains with an application to multigrid theory. Math. Comp., 64:1359-1365, 1995. MR 95m:46042

7. J. H. Bramble and S. R. Hilbert. Estimation of linear functionals on Sobolev spaces with applications to Fourier transforms and spline interpolation. SIAM J. Numer. Anal., 7:113124, 1970. MR 41:7819

8. J. H. Bramble, J. Pasciak and P. S. Vassilevski. Computational scales of Sobolev norms with applications to preconditioning. Math. Comp., 69:463-480, 2000. MR 2000k:65088

9. J. H. Bramble, J. Pasciak and J.Xu. Parallel multilevel preconditioners. Math. Comp., 55:1-22, 1990. MR 90k:65170

10. J. H. Bramble and X. Zhang. The analysis of multigrid methods, in: Handbook for Numerical Analysis, Vol. VII, 173-415, P. Ciarlet and J.L. Lions, eds., North Holland, Amsterdam, 2000. MR 2001m:65183

11. S. Brenner and L.R. Scott. The Mathematical Theory of Finite Element Methods. SpringerVerlag, New York, 1994. MR 95f:65001

12. P. G. Ciarlet. The Finite Element Method for Elliptic Problems. North Holland, Amsterdam, 1978. MR 58:25001

13. M. Dauge. Elliptic Boundary Value Problems on Corner Domains. Lecture Notes in Mathematics 1341. Springer-Verlag, Berlin, 1988. MR 91a:35078

14. M. Dobrowolski. Numerical Approximation of Elliptic Interface and Corner Problems. Rheinischen Friedrich-Wilhelms-Universität, Bonn,1981.

15. P. Grisvard. Elliptic Problems in Nonsmooth Domains. Pitman, Boston, 1985. MR 86m:35044

16. P. Grisvard. Singularities in Boundary Value Problems. Masson, Paris, 1992. MR 93h:35004

17. P. Grisvard. Caracterisation de quelques espaces d'interpolation. Arc. Rat. Mech. Anal. 25:4063, 1967. MR 35:4718

18. R. B. Kellogg. Interpolation between subspaces of a Hilbert space, Technical note BN-719. Institute for Fluid Dynamics and Applied Mathematics, University of Maryland, College Park, 1971.

19. V. Kondratiev. Boundary value problems for elliptic equations in domains with conical or angular points. Trans. Moscow Math. Soc., 16:227-313, 1967. MR 37:1777

20. V. A. Kozlov, V. G. Mazya and J. Rossmann. Elliptic Boundary Value Problems in Domains with Point Singularities. American Mathematical Society, Mathematical Surveys and Monographs, vol. 52, 1997. MR 98f:35038

21. J. L. Lions and E. Magenes. Non-homogeneous Boundary Value Problems and Applications, I. Springer-Verlag, New York, 1972. MR 50:2670

22. J. L. Lions and P. Peetre. Sur une classe d'espaces d'interpolation. Institut des Hautes Etudes Scientifique. Publ.Math., 19:5-68, 1964. MR 29:2627

23. S. A. Nazarov and B. A. Plamenevsky. Elliptic Problems in Domains with Piecewise Smooth Boundaries. Expositions in Mathematics, vol. 13, de Gruyter, New York, 1994. MR 95h:35001

24. J. Nečas. Les Methodes Directes en Theorie des Equations Elliptiques. Academia, Prague, 1967. MR 37:3168

25. P. Oswald. Multilevel Finite Element Approximation. B. G. Teubner, Stuttgart, 1994. MR 95k:65110

26. L. Wahlbin. On the sharpness of certain local estimates for $H_{0}^{1}$ projections into finite element spaces: In fluence of a reentrant corner. Math. Comp., 42:1-8, 1984. MR 86b:65129 
27. J. Xu. Iterative methods by space decomposition and subspace correction. SIAM Review, 34:581-613, December 1992. MR 93k:65029

28. K. Yosida. Lectures on Differential and Integral Equations. Dover Publications, Inc., New York, 1991. MR 92a:34002

Dept. of Mathematics, The Pennsylvania State University, University Park, PennSYLVANIA 16802

E-mail address: bacuta@math.psu.edu

Department of Mathematics, Texas A \& M University, College Station, Texas 77843

E-mail address: bramble@math.tamu.edu

Department of Mathematics, The Pennsylvania State University, University Park, PenNSYlVANia 16802

E-mail address: xu@math.psu.edu 